\title{
Comparing Inductive and Deductive Modeling of Land Use Decisions: Principles, a Model and an Illustration from the Philippines
}

\author{
Koen P. Overmars • Wouter T. de Groot • \\ Marco G. A. Huigen
}

Published online: 24 January 2007

(C) Springer Science + Business Media, LLC 2007

\begin{abstract}
Understanding the causes of land use change is of great importance for issues of tropical deforestation, agricultural development and biodiversity conservation. Many quantitative studies, therefore, aim to link land use change to its causal 'driving forces.' The epistemology of virtually all these studies is inductive, searching for correlations within relatively large, sometimes spatially explicit, datasets. This can be sound science but we here aim to exemplify that there is also scope for more deductive approaches that test a pre-defined explanatory theory. The paper first introduces the principles and merits of inductive and more deductive types of land use modeling. It then presents one integrated causal model that is subsequently specified to predict land use in an area in northeastern Philippines in a deductive manner, and tested against the observed land use in that area. The same set of land use data is also used in an inductive (multinomial regression) approach. With a goodness-of-prediction of $70 \%$ of the deductive model and a goodness-of-fit of $77 \%$ of the inductive model, both perform equally well, statistically. Because the deductive model explicitly contains not only the causal factors but also the causal mechanisms that explain land use, the deductive model then provides a more truly causal, as well as more theory-connected, understanding of land use.
\end{abstract}

K. P. Overmars · W. T. de Groot • M. G. A. Huigen Institute of Environmental Sciences (CML), Leiden University, Leiden, The Netherlands

K. P. Overmars $(\square)$

Department of Environmental Sciences, Wageningen University, P.O. Box 37, 6700 AA Wageningen, The Netherlands e-mail: Koen.Overmars@wur.nl

W. T. de Groot • M. G. A. Huigen

University of Nijmegen,

Nijmegen, The Netherlands
This provides land use scholarship with an invitation to add more deductive (theory-driven and theory-building) daring to its methodological repertoire.

Key words Philippines · land use - modeling · induction · deduction $\cdot$ action-in-context $\cdot$ actor-based model . multinomial logistic regression

\section{Introduction}

The face of the earth is rapidly changing, with great consequences for rural livelihoods, biodiversity conservation, urban quality of life and the global climate. Understanding land use change is therefore a matter of obvious import and urgency.

Land use change is the result of the complex interplay of underlying causal factors, usually referred to as 'driving forces,' that may vary across scales and organizational levels, that may work directly or through longer causal routes and that may be associated with different societal and scientific realms, such as markets, policies, demography, culture and biophysical factors. How can such complexity be handled scientifically? One approach is to focus on only one or a few factors, and accept that explanations can only be partial. Generally, however, land use scientists desire a more 'integrated' (multi-factor) analysis. As shown, for instance, in overviews of Walker et al. (2002) and Verburg et al. (2004b), the great majority of the present-day blooming of quantitative integrated (multi-factor) studies of land use change follows an inductive approach, sometimes guided by theory but without testing the theory as such. In the present paper, we make a case that the present state of the art allows us to perform integrated research and yet use a more deductive epistemology, and that this option, in 
interaction with inductive work, will enhance causal insight and cumulative scientific progress in land use science. We aim to strengthen our case by showing and discussing the performance of a deductive and an inductive approach, applied parallel to each other to explain land use in a single example region.

"Inductive Versus Deductive Modelling" discusses the principles and merits of inductive and deductive approaches to land use science. "The Action-in-Context Framework and Decision Model" is devoted to the structure of the theoretical model for our case study. After "Materials and Methods," "Qualitative Description of the Deductive Model" formalizes the deductive model for our example region and "Quantifying the Deductive Model" quantifies the model. "Model Results" describes the results of the deductive model as well as those of a (inductive) multinomial regression model. The ensuing discussion shows the value of the deductive modeling approach within a range of approaches from inductive to deductive.

\section{Inductive Versus Deductive Modelling}

Deductive and Inductive Epistemology in Integrated Land Use Explanations

For most of us, the "empirical cycle" must have been the first concept taught in lectures about how science proceeds. First there is a theory; then a concrete predictive hypothesis is deduced from that theory. Then this hypothesis is tested in the real world and with that result, the theory is either falsified or strengthened. This, in short, is deductive epistemology. Contrasting with this approach, inductive methodology begins with observations of reality and then tries to find regularities in these data. This regularity is then declared to be a general pattern (a model, a theory), which again can be tested in practice.

The present paper does not find fault in this basic epistemological scheme. We do, however, think that for a proper understanding of how land use science proceeds in practice, it is necessary to define a number of methodological positions that lie in-between the deductive and inductive extremes. On the one hand, there is extreme deduction (Popper, 1963) in which the empirical cycle is followed strictly and theory falsification rather than verification is seen as the key to progress. On the other hand, there is extreme induction, in which the researcher aims to find patterns in large datasets without any theoretical guide. Both extremes have their advantages in some cases, depending on the availability of theory. Both have disadvantages too, however. In the social and economic sciences, extreme deductivism would lead to an endless rejection of theories because simply none of them is able to grasp the full complexity of the system described. Extreme inductivism, on the other hand, leads to an immense amount of correlations that cannot be interpreted as causes and never accumulate into a coherent theory.

In most research practice, researchers find a less daunting solution by adopting a position, usually implicit and led by disciplinary traditions, somewhere on the continuum between extreme induction and extreme deduction. For the present paper and including the two extremes, we may define six of these positions. We concentrate here mainly on quantitative work.

1. Extreme induction. The first case is that a researcher has no model or theory at all. The only methodology available is extreme induction in which the researcher attacks large datasets, basically 'correlating everything with everything else' in order to see if any patterns may be found. This is the extreme of data mining, or "knowledge discovery in databases" (Liao, 2003).

2. Unstructured factors induction. Under this term we subsume all research approaches that apply a broad conceptual framework of some kind, usually derived from common sense or literature overview, in order to specify a usually long list of factors or proxies that are candidate to help explain land use or land use change. (Alternatively, some kind of theory may be invoked as well, e.g. Nelson et al. (2004), saying that land users choose for the most profitable land use, but then these theories are in fact only serving as a broad conceptual framework.) The studies then leave it to the procedures of statistical inference to find the correlations between these variables. Characteristically, these studies do not end with a discussion of theoretical perspectives but with a discussion of the significance of correlation coefficients and such like in the specific case studied. Many land use change studies fall into this category (e.g. Serneels and Lambin, 2001; Overmars and Verburg, 2005).

3. Theory-guided factors induction. This term denotes studies that take an explicit theory of land use change as point of departure to critically specify a theoryconnected (usually shorter) list of explanatory variables. Strictly speaking, this list is still unstructured without specification of how the variables are supposed to interact. On the other hand, the variables are not simply 'candidates' that are dropped if they do not contribute to the explanation. If they do not contribute, something is 'wrong' with the theory or its interpretation, which needs to be discussed. One quantitative example is in Perz and Walker (2002), focusing on secondary forest growth in Amazonia in connection with Chayanovian theory. Interesting results have also been reached in a more qualitative manner, exemplified 
by Ostrom (1990) who arrived at her well-known conditions for successful common property management by a stepwise induction of case studies. Characteristic for all studies of theory-guided induction is that the relevance of the results is wider than those of type 1 and type 2 studies. Guided by theory, induction can become theory building.

4. Imposed theory structure. The next rung on the induction/deduction ladder is formed by studies that impose not only theory-guided factors but also a theory-guided structure (the 'behavioral statements', as Walker (2004) says) on reality before multiple regression is applied in order to induce the parameters within that structure. According to Elster (1989), it is only then that true explanation comes within reach, because true explanation requires insight not only in the factors but also in the mechanisms. If our theory is, for example, that people only choose for a land use type to the extent that this land use type is both culturally appropriate and profitable, our model structure would look like Liebig's law on plant growth, which states that plants do not respond to nutrients (factors) additionally but only to the one factor 'in the minimum'; see Tadepally (1999) for an example on collective motivation, collective social capital and the success of NGO efforts in irrigation system rehabilitation. The 'imposed structure' approach can also be used in a more qualitative style. A theory is then used to 'tell the story' of a specific case of land use change, as do, for instance, Walker and Solecki (2004) and De Groot (1999), who apply dynamic versions of Thünian theory to explain land use history.

5. Imposed theory. A purely deductive approach is reached when a land use theory is specified for a real world case in terms of both structure and parameters, and the land use thus predicted is tested against real land use. It is only then that true prediction, hence true deduction, is possible. The case study of the present paper will be an example.

6. Extreme deduction. We keep the 'Popperian' extreme separate here because in step 5, the model and the data gathering are not geared towards falsification and neither need theories to be dropped if they do not work adequately yet.

A few technical remarks are in order here. First, induction, deduction and the continuum between them, even though central tenets of epistemology, do not cover the full spectrum of scientific methodology. Creative inference ('abduction') and the heuristic concepts of 'event ecology' (Vayda and Walters, 1999) are cases in point. Second, we may note that the six rungs of the induction/deduction ladder are naturally not the only possible ones. Researchers may also find intermediate and mixed positions, or work sequentially, for instance. We do not go into these issues here, however, and regard the listing as good enough to indicate what we mean when saying that land use studies could or should become 'more deductive'.

\section{Could Land Use Explanation Studies Become More Deductive?}

Overlooking the field of explanatory land use studies, we find many examples of unstructured factors induction. Theory-guided factors induction is present in much smaller numbers. Imposition of theory structure is virtually nonexistent. This may have historical and cultural reasons. To begin with, strong theories that may be tested are simply not massively present in any young scientific field. Furthermore, theories and deduction are not really en vogue in post-modern times (they are top-down, they turn a blind eye to the multiple complexities and voices of social realities, etc.). And finally, the attraction that land use studies appear to have had to econometrists and GIS-based geographic data technology may block growth towards more deductive, theoryguided work.

In our opinion, explanatory land use studies could become more deductive. We do have land use theories to use and test, if only simple ones, for example neo-Malthusian theory, neoBoserupian theory and neo-Thünian theory. And we have more general explanatory theories waiting to be applied and tested on land use situations, such as rational choice theory, cultural theory, theories of collective action and common property management. Furthermore, much knowledge has accumulated in datasets for inductive analyses, which may be conceptually re-used in more deductive ways. In the present paper, our example shows that nothing conceptually difficult is at stake here.

\section{Should Land Use Explanation Studies Become More deductive?}

There are two main advantages of using deductive methods. First, deduction yields the intrinsically better proof of causality, i.e. true explanation. Let us take Nelson et al. (2004) as an example. Their causal model is that on each site, the most profitable crop is grown. However, this is not tested as such because, as Nelson et al. say, profitability is not measurable. Instead, factors such as slope are used as explanatory variables. If, say, maize is found to be associated with medium slopes, would that be because of its relative profitability there? It could also be that traditions do not allow maize elsewhere, or because of risk aversion. The slopes/maize correlation does not establish any causality. If, however, Nelson et al. had calculated the 
spatially explicit profitability of crops (based on prices, distance to road, slope etc.), then would had set the model to predict crop distribution on the basis of highest profitability, and then would have found the model predicting maize on medium slopes, a strong proof of causality would have been delivered, because not only the factors but also the mechanism would have been tested.

The second benefit of a more deductive approach is that it better facilitates the accumulation of insight on the level of the discipline as a whole. Referring back again to the example of Nelson et al. (2004), the outcomes of rung 2 studies are basically the strengths-of-correlation between land use and a long list of independent variables. Conclusions then necessarily tend to remain largely stuck on that level, e.g. that maize tends to converge on certain slopes. In order to reach some degree of generalization, such studies then have to wait until enough of them have accumulated to themselves become data in a meta-analysis such as that of Geist and Lambin (2002) who, characteristically for an inductive approach in the meta-analysis of inductive studies, come up with a generalized and regionally patterned listing of proximate factors and underlying driving forces of tropical deforestation. Obviously useful as this may be, more progress would be made if not only the incidental metaanalyst but also the researchers themselves, in their own studies, were able to participate in a permanent intertrade of generalization. This can be achieved if these studies were more deductive, i.e. more theory-led. Theory-led work, feeding back into theory, leads to theory building.

In all this, we assume that empirically-based theories are good to have. In other words, we assume that land use scientists do not become addicted to theories, especially their own, to a degree that theories begin to block entry for the surprises of reality (Vayda, 1983) or become objects of counterproductive controversy (Brox, 1990).

Model Choice and Levels-of-deductivism of this Paper

As stated, the objective of the present paper is to expound and illustrate the deduction/induction dimension for integrated land use explanation. For illustrating the deductive approach, we have chosen to test a broad model that is able to take up all factors that should be comprised in an integrated approach, hence including cultural, economic and biophysical data. The model has been taken from De Groot (1992), and may be characterized as broad rational choice. For the inductive approach a multinomial logistic regression model is applied. As for the positions on the deduction ladder, we have chosen to compare an 'unstructured factors induction' (rung 2) with a fully deductive approach, 'imposed theory' (rung 5), hence a true test without any subsequent fitting on the dataset. In the remainder of this paper these two approaches are referred to as the inductive and deductive approach or model, respectively. We put all emphasis on the comparison and not on the cultural or land use intricacies of the study area.

\section{The Action-in-Context Framework and Decision Model}

Action-in-Context (AiC) (De Groot, 1992) is a framework designed for the explanation of human actions, especially in the environmental field. Based on the concept of progressive contextualization (Vayda, 1983), the idea of AiC is to start out from the action to be explained, then identify the (individual or collective) actors directly causing this action, then identify the range of options available to these 'primary' actors and the motivations attached to these options, and then identify other ('secondary') actors and factors influencing these options and motivations, thereby putting the action in its relevant causal context without a priori bias towards any scientific discipline (Vayda and Walters, 1999). With that, $\mathrm{AiC}$ is a fully actor-based framework, which is a logical choice for explanatory work because actors, not systems, are the social entities that cause change directly. ${ }^{1} \mathrm{AiC}$ may be used as a framework to guide the research process, but can also be used as a template for models. These models can be, for example, detailed multi-agent models that model individual agents (Huigen, 2004), or models that explain the choices of a smaller number of large actor categories. The latter is of course much simpler to implement and the way we will proceed in this study.

Action-in-Context has four interconnected components. (1) The first is an often repeated "core element," comprising of the action, the actor, his options and his motivations. In Elster (1989), the latter two are called "opportunities" and "desires" but the structure is of the same simplicity: in order to act, people must have both the capacity and the will to do so. The other components of AiC are elaborations of the core element. (2) The "actors field" is an aspect of $\mathrm{AiC}$ that describes the chains of social influence (causality, power) that run from the primary actors outward to other actors. Moving from primary to secondary actors and further is the actor-based way of moving from proximate factors to underlying drivers sensua Geist and Lambin (2002). (3) The next component in AiC may be mixed freely with the preceding one and consists of a "deeper analysis" of the options and motivations of selected actors, distinguishing, inter alia, between elements of knowledge,

\footnotetext{
${ }^{1}$ Also Blaikie (1985) has this basic notion of explanation by putting actions of actors in context, but his contexts are conceptualized as systems rather than other actors. In AiC, explanations may reach up to the global level but this level is then still present as actors.
} 
resources, economic merit and culture. Figure 1 is AiC's broadly rational decision model designed to support this step, which will be discussed in detail below. The deeper analysis is a second way to connect proximate factors to underlying culture and structure. (4) The final component of AiC is called the "actor model," which defines how the actor evaluates the options and motivations to come to his decision. In qualitative research, the actor model can often remain implicit. In such cases, the researcher 'puts himself in the place of the actor' (Vayda, 1983). Another actor model is rational choice, which is to say that the actor chooses for the option of maximum merit - or utility, or profit, - in which the definition of these terms marks the difference between narrow and broad rational choice. Specific for AiC is that it also offers an actor model for cases where a deeper reflection on the logic of human decision-making is warranted. This actor model distinguishes among three 'moral domains' of reasoning: homo economicus (i.e. rational choice), homo honoris and homo communalis (or 'ethics of care') (De Groot, 1992).

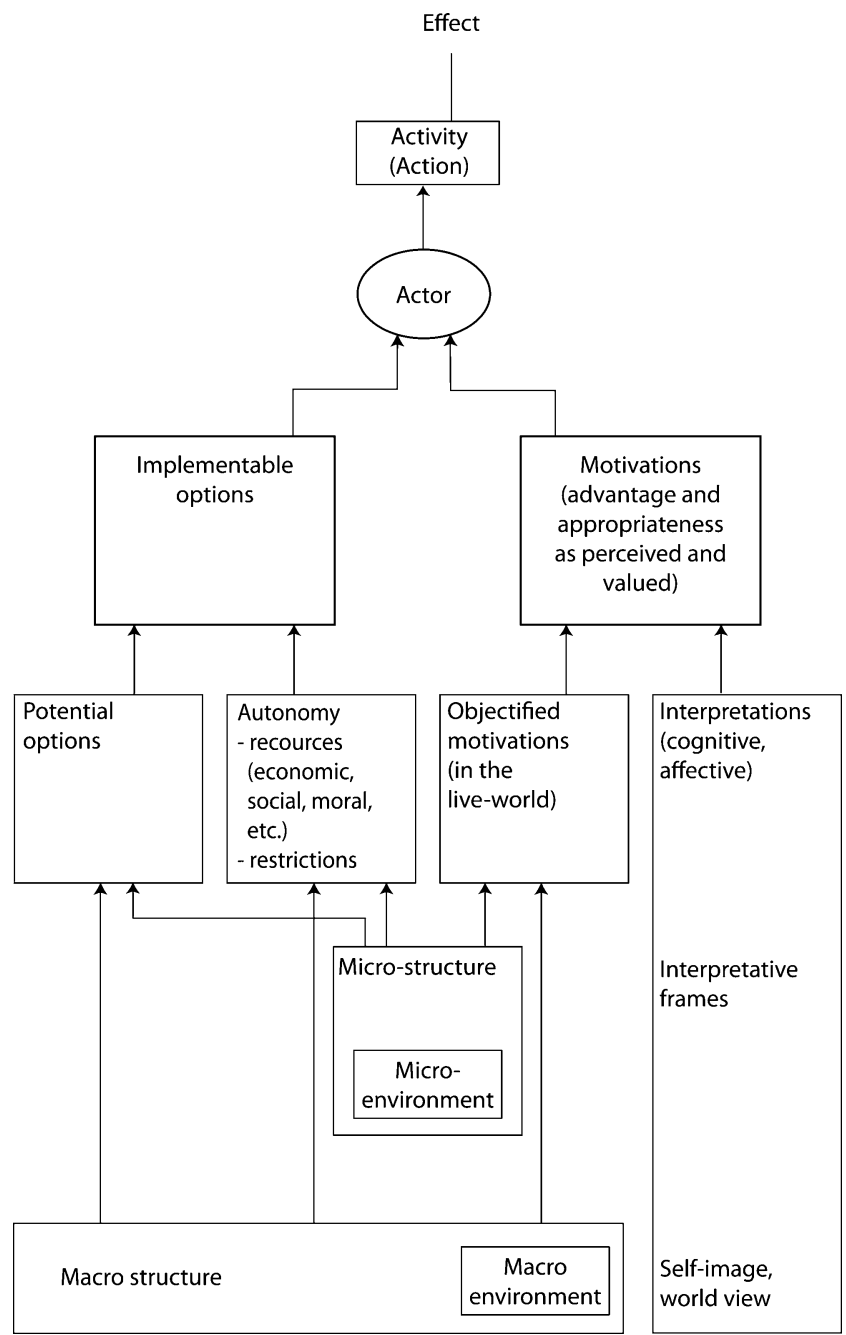

Fig. 1 The decision model structure of AiC.
Action-in-Context may be applied in many forms, in full or partially, formal or informal, as a heuristic tool for guiding fieldwork or as a static model. See, for instance, Van den Top (1998) for a qualitative application on tropical deforestation. In the present paper, we will use and test a fully quantified version (i.e. structure and all parameters) of the decision model of the deeper analysis (Fig. 1).

In Fig. 1, all arrows stand for causal relations. The top layers of the figure repeat the core element of AiC - the options are now specified as "implementable" options and the motivations are the options' merits ("advantage and appropriateness") "as perceived and valued" by the actor. These specifications facilitate the definitions on the next lower level, which is the one of most interest here, where the implementable options are seen to result from the actor's "potential options" and "autonomy". Potential options are defined as everything the actor could do if he were infinitely autonomous (rich, powerful). Basically, they are all options that the actor knows to be possible. "Autonomy" is capacity-to-implement, and is defined as the sum of all resources the actor can access (economic capital, private social capital, cultural capital, entitlements to common goods, etc.), taking into account possible restrictions (e.g. zoning regulations). Potential options and autonomy together determine the implementable options. Figure 1 does not specify the structure of this joint causality but we may note that it cannot be some simple form of addition; just adding potential options does not automatically add to implementable options (let alone change actions), and neither does just adding to the actor's autonomy ('empowerment'). The case study of this paper shows one way of modeling this.

Motivations are the merits of the options. In Fig. 1, the motivations "as perceived and valued" are separated into "objectified motivations" and their "interpretations." Objectified motivations are all those that may easily be quantified, such as economic cost and benefits, time expenditure, risk probabilities, caloric value of food, etc. - factors that microeconomists and farming system analysts feel at home with. Interpretations, on the other hand, are all those factors that give weight, coherence, shape and color to the objectified motivations. Note that this way the interpretations are set as somehow multipliers of the objectified motivations rather than a 'filter' between actor and reality; psychology and culture add life to the actor, so to speak. Deeper down in the figure (but without causal arrows, indicating that the relationship is difficult to quantify) these interpretations are supposed to rise out of broader "interpretative frames" and "self-image/worldview." One example is the actor's image of what it is to be a good farmer (Zuiderwijk, 1998).

In Fig. 1 furthermore, the third-layer elements are supposed to arise out of the actor's micro-structure (defined 
as all structures, social and physical, where the actor makes a difference) and macro-structure. Since these relationships do not play a role in our quantified model, we do not go into them here.

Overall, Fig. 1 is obviously not something special but rather designed as the reverse. It aims to overarch and coherently integrate all elements of broad rational choice theory, including cultural elements, the 'capitals' of Bebbington (1999) and so on, and remain close to the models of social psychology (albeit dropping the cumbersome intervening variable of 'attitudes'). Roughly then, many disciplinary focal points are included in the model: the options of agronomy and forestry, the objectified motivations of economics, the culture of anthropology, the capitals (autonomy) of development studies, the environment of geographers, and so on. Thus, the model facilitates explanatory work without preoccupation towards any specific discipline.

\section{Materials and Methods}

\section{Study Area}

The study area is situated in Cagayan Valley in the northeastern part of the island Luzon, the Philippines (Fig. 2), and includes 13 villages in the municipality of San Mariano, in the province of Isabela, comprising approximately $230 \mathrm{~km}^{2}$. It is situated between the town of San Mariano in the west and the forested mountains of the Sierra Madre in the east.

The population is approximately 16,500 persons (about 3,150 households) of various ethnic groups. The Ilocano, Ibanag and Ifugao are all migrants or descendents of migrants that came to the area from the 1900 s onwards,

Fig. 2 Study area.



and the Kalinga and Agta are the indigenous inhabitants. Before immigration started, the area was completely forested with tropical lowland forest. At present, the study area shows a clear land use gradient ranging from intensive agriculture, with wet rice and yellow corn, near San Mariano via a scattered pattern of wet rice, yellow corn, banana, grasses, and (fruit) trees in the foothills to residual and primary forest in the eastern part.

\section{Data Collection}

We conducted household-level interviews between June and November 2002, using a structured questionnaire, to collect data on land use and its explanatory variables. Selection of households to be interviewed was based on systematic random sampling of every twentieth household. A total of 151 households were interviewed. Records containing missing values and households without any land were excluded from the dataset. In total 114 households were included in the analysis. These households manage 272 fields. These data were used to fit the multinomial regression model of the inductive approach and to validate both the inductive and the deductive model. Table I shows a selection of the dataset consisting of those variables that turned out to be relevant in this case study.

Besides the questionnaires, semistructured interviews were held with farmers and key-actors. If possible, these interviews were held with a group of people to enable discussion and verification. These interviews dealt, inter alia, with the motivations of the people to grow one crop or another. These semi-structured interviews were guided by the AiC framework, which was used for progressive contextualization (Vayda, 1983) in this stage. The information from these semi-structured interviews was used to qualitatively describe the processes in the area as well as to quan-

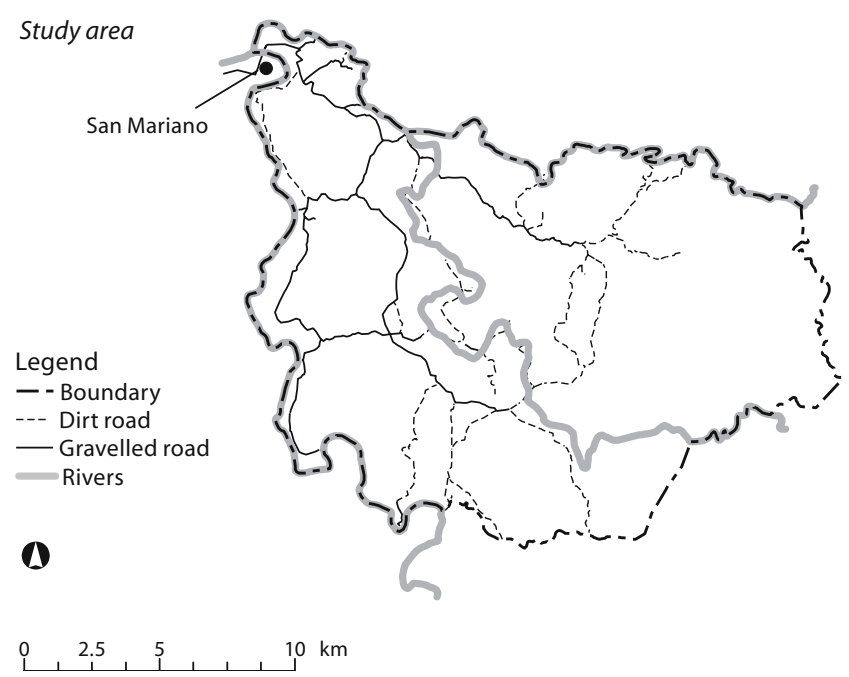


tify the decision model and to determine the calculi to relate the elements of this model.

\section{Analysis}

For the inductive approach to predict the land use on a field we applied multinomial logistic regression, which is regularly used in land use analysis (e.g. Nelson et al., 2001; Müller and Zeller, 2002). Multinomial logistic regression extends the possibilities of logistic regression by allowing for more than two categories in the dependent variable. In this case four land use categories are included. The parameter estimates are calculated simultaneously and the probabilities of the different land use categories add up to one. The final prediction is the land use with the highest probability. In the multinomial model, the estimated parameters are to be interpreted in relation to one of the categories of the dependent variable, which serves as reference category.
In the multinomial regression the following variables were taken into account: slope, ethnicity, municipality of origin, presence of creek, plot distance, transportation cost, average age of the household heads and tenure (Table I). The approach follows the rung 2 level of the deduction/ inductive ladder presented previously: selection of the factors was inspired by several (disciplinary) land use theories, prior studies (Overmars and Verburg, 2005) and field knowledge. These theories are not tested as such, but their factors are used in the regression analysis.

For the deductive approach, the causal model we applied is a quantification of Fig. 1. As actor model we used broad rational choice, saying that the actor chooses for the implementable option of maximum merit. In the next section, a qualitative description of the case is provided following the structure of the model. The subsequent section then quantifies this model. The model was used to predict the probability of the occurrence of the land use types. We used the same set of explanatory factors as in the

Table I Description and Descriptive Statistics of the Variables of the Household Survey $(n=272)$

\begin{tabular}{|c|c|c|c|c|c|}
\hline Variable name & Description & Mean & $\begin{array}{l}\text { St. } \\
\text { Dev. }\end{array}$ & $\begin{array}{l}\text { Incl. in ded. } \\
\text { model }\end{array}$ & $\begin{array}{l}\text { Incl. in ind. } \\
\text { model }\end{array}$ \\
\hline \multicolumn{6}{|l|}{ Dependent variables } \\
\hline Yellow corn & 1 if yellow corn, 0 otherwise & 0.58 & & $\mathrm{Y}$ & $\mathrm{N}$ \\
\hline Wet rice & 1 if wet rice, 0 otherwise & 0.13 & & $\mathrm{Y}$ & $\mathrm{N}$ \\
\hline Banana & 1 if banana, 0 otherwise & 0.24 & & $\mathrm{Y}$ & $\mathrm{N}$ \\
\hline Fruit trees & 1 if fruit trees, 0 otherwise & 0.05 & & $\mathrm{Y}$ & $\mathrm{N}$ \\
\hline Land use & $\begin{array}{l}\text { yellow corn (4), wet rice (1), banana (2) and fruit (3) } \\
\text { (nominal) }\end{array}$ & & & $\mathrm{N}$ & $\mathrm{Y}$ \\
\hline \multicolumn{6}{|l|}{ Independent variables } \\
\hline Slope1 & 1 flat slope, 0 otherwise & 0.38 & & $\mathrm{Y}$ & $\mathrm{N}$ \\
\hline Slope2 & 1 flat to rolling/moderate slope, 0 otherwise & 0.23 & & $\mathrm{Y}$ & $\mathrm{N}$ \\
\hline Slope3 & 1 rolling/moderate slope, 0 otherwise & 0.28 & & $\mathrm{Y}$ & $\mathrm{N}$ \\
\hline Slope4 & $1 \mathrm{rolling} / \mathrm{moderate}$ to steep/hilly slope, 0 otherwise & 0.08 & & $\mathrm{Y}$ & $\mathrm{N}$ \\
\hline Slope 5 & 1 steep/hilly slope, 0 otherwise & 0.03 & & $\mathrm{Y}$ & $\mathrm{N}$ \\
\hline Slope & 1 (flat) to 5 (steep), ordinal & & & $\mathrm{N}$ & $\mathrm{Y}$ \\
\hline Ethnicity Ifugao & 1 if male household head is Ifugao, 0 otherwise & 0.10 & & $\mathrm{Y}$ & $\mathrm{Y}$ \\
\hline Ethnicity Ibanag & 1 if male household head is Ibanag, 0 otherwise & 0.30 & & $\mathrm{Y}$ & $\mathrm{N}$ \\
\hline Ethnicity Ilocano & $\begin{array}{l}1 \text { if male household head is Ilocano (or Tagalog speaking), } \\
0 \text { otherwise }\end{array}$ & 0.55 & & $\mathrm{Y}$ & $\mathrm{Y}$ \\
\hline Ethnicity Kalinga & 1 if male household head is Kalinga, 0 otherwise & 0.03 & & $\mathrm{Y}$ & $\mathrm{N}$ \\
\hline Ethnicity Other & $\begin{array}{l}1 \text { if male household head is other than Ifugao,Ibanag, Ilocano } \\
\text { or Kalinga }\end{array}$ & 0.02 & & $\mathrm{Y}$ & $\mathrm{N}$ \\
\hline $\begin{array}{l}\text { Municipality of } \\
\text { origin }\end{array}$ & $\begin{array}{l}1 \text { if male or female head is born in San Mariano, } 2 \text { if both, } \\
0 \text { otherwise }\end{array}$ & 1.17 & & $\mathrm{Y}$ & $\mathrm{Y}$ \\
\hline Creek & $\begin{array}{l}1 \text { if there is a creek or spring trough or bordering the plot, } \\
0 \text { otherwise }\end{array}$ & 0.58 & & $\mathrm{Y}$ & $\mathrm{Y}$ \\
\hline Plot distance & Travel time to the plot (min) & 23.81 & 33.50 & $\mathrm{Y}$ & $\mathrm{Y}$ \\
\hline $\begin{array}{l}\text { Transportation } \\
\text { cost }\end{array}$ & $\begin{array}{l}\text { Cost to transport a bag of corn from the house to San Mariano } \\
\text { (pesos) }\end{array}$ & 23.85 & 12.49 & $\mathrm{Y}$ & $\mathrm{Y}$ \\
\hline Average age & Average age of household heads (years) & 42.71 & 13.07 & $\mathrm{Y}$ & $\mathrm{Y}$ \\
\hline Tenure tenant & 1 if the household is tenant of the plot, 0 otherwise & 0.19 & & $\mathrm{Y}$ & $\mathrm{Y}$ \\
\hline Tenure SIFMA & 1 if the plot is has SIFMA tenure, 0 otherwise & 0.06 & & $\mathrm{Y}$ & $\mathrm{Y}$ \\
\hline
\end{tabular}


multinomial model, together with some additional constants like maximum benefit and investments.

Since the inductive (regression) model and the deductive (AiC) model describe the land use system in the same area and use the same variables, the performance of the models can easily be compared. The performance was calculated using cross-tables (prediction matrix). The outcomes are a goodness-of-fit of the inductive model and a goodness-ofprediction of the deductive model. The cross-tables show the observed category against the modeled category of land use.

\section{Qualitative Description of the Deductive Model}

Actors and Actions

Ninety percent of the households in the study area have a piece of land to cultivate. Farming is the main source of income of $80 \%$ of the households and the second source of income for $10 \%$ of the households. Besides this, most people work also as laborers for other farmers, which provides them with additional income. The actors considered in the analysis are those households that have control over a piece of land that they can possibly cultivate. They will be called farmers in the remainder of the paper.

The analysis focuses on the decision-making on agricultural land use types. However, the possibilities of making a living in the area are broader than agriculture alone. Besides options that do not involve land (e.g. carpenter, storekeeper, driver), the people in the area also have other land use options such as small-scale logging and collecting nontimber forest products. Neither of these option categories are considered in this study. Hence, the research question is why farmers cultivate a certain crop at a certain location. The area that the farmer cultivates per crop is not subject of analysis.

\section{Potential Options}

To construct a list of potential land use options we could include, for example, all crops grown in the region over the last 30 years. Considering our research objective, which is a methodological comparison rather than location specific detail, we chose to include only wet rice, yellow corn, banana and fruit trees, which currently account for $92 \%$ of the observed fields.

\section{Autonomy}

The level of autonomy determines if a potential option can be implemented or not. Autonomy consists of two elements: resources and (absence of) restrictions. If the farmer is a tenant of the land he cultivates, the landowner often decides what the tenant should cultivate, which is most often yellow corn. For the cultivation of wet rice two restrictions were added to the model: presence of a creek and the farmers' ethnicity. Wet rice cultivation is restricted to Ifugao and Ilocano because, generally speaking, they have better skills and knowledge in constructing rice fields and rice terraces and cultivating wet rice. The Ibanag, originally lowlanders of the Cagayan valley, have a long tradition in corn cultivation but many have less knowledge of wet rice cultivation (Romero, personal communication). The presence of a creek on or near the plot is important for the cultivation of wet rice.

The other element of autonomy consists of the resources of a farmer. In this case study, resources are considered to be necessary to make the initial investments to start a new land use type, like clearing a forested area for corn cultivation or constructing a rice terrace. If the resources are sufficient, the land use type is an implementable option, and therefore they function as a threshold. They are built up of two components: basic investments and, for rice only, additional investments dependent on slope. In our model, the resources are composed of the level of assistance, the possibility to obtain credit to buy inputs for a crop and participation in the Socialized Integrated Forest Management Agreement (SIFMA) program, which together should be sufficient for the initial investments for a specific land use type.

The resource 'level of assistance' is composed of the factor municipality of origin and a factor proportional to the average age of the household heads. The municipality of origin of the household heads is considered to be indicative of the size of a household's social network (roughly: private social capital); assuming that people born in San Mariano have more relatives and friends nearby to help out than people from outside the municipality (Moonen, 2002). Ifugao were considered to have assistance from relatives even when they are not from San Mariano, because often they migrate after invitations from relatives or friends and cluster together. Also a higher age is considered to be indicative or a larger network to organize labor (children, relatives).

Another way to meet the necessary investments is to borrow money. In the research area credit is almost exclusively provided for yellow corn. Other sources of capital to make investments for other crops are hardly available, which actually restricts farmers in their options.

The last resource is participation in the SIFMA program, which provides tenurial security and assistance in starting an agro-forestry plantation and therefore applies to the land use type fruit trees. Governmental and non-governmental organizations encourage farmers to invest in agro-forestry systems on sloping lands. Farmers that were awarded a SIFMA contract can receive free fruit tree seedlings to be planted on their SIFMA lot, covering a part of the high initial investment costs. 


\section{Objectified Motivations}

Motivations are composed of objectified motivations and interpretations. In this study the objectified motivations are considered to be the net economic benefit from 1 ha of a land use type at the moment the product is sold in San Mariano (yellow corn, banana and fruit trees) or consumed (rice). The net benefit is defined as the maximum benefit under ideal climatological and biophysical conditions (no droughts or typhoons; flat area with a good soil) for an average price, multiplied by a yield-reducing factor depending on slope (for yellow corn) and a yield-reducing factor depending on risks lowered with the transport cost. The maximum benefit is considered to be the same at all locations in the study area.

Bananas can grow in every landscape position, unless soil drainage is very bad (Valmayor et al., 1990). Many of the drawbacks that corn has on steep slopes do not apply to banana. Banana cultivation does not involve tillage. Bananas are renewed only once every 5-15 years. The productivity of banana is the same on steep slopes and flat areas. So, slope does not influence the motivation towards growing banana. On the contrary, many farmers plant bananas to prevent soil erosion on steep slopes.

Transportation cost is defined as the cost to transport the product from a farmer's home to the market. In this study transportation costs apply to yellow corn, banana and fruits. Rice is used for household consumption or sold in the neighborhood. Additionally, the distance from the plot to the residence of the farmer is taken into account. Plots far from the farmer's village take more travel time. Moreover, fields that are far away are at more risk from damage by fire, water buffalo or people.

Interpretations

The objectified motivations are adjusted to the interpretation of the individual farmer. In this model interpretations are simplified to crop preferences of the different ethnic groups. As said before, the Ifugao and Ilocano have a tradition of wet rice cultivation whereas the Ibanag have a tradition of corn cultivation and not wet rice cultivation. This is reflected in their preference for corn and rice. The objectified motivations are combined with the interpretations to become the motivations "as perceived and valued." This may cause people of different ethnicities to choose a different land use option even if the objectified motivations are equal.

\section{Quantifying the Deductive Model}

Based on the fieldwork and the qualitative analysis in the previous section, the model of Fig. 1 is quantified as follows.
The core of the model is that the predicted land use is the implementable land use option with the highest motivation (Eq. 1). Starting with the options side of the model, Eq. 2 shows that the implementable options are composed of potential options and autonomy. The potential options are yellow corn, wet rice, banana and fruit trees. Autonomy (Eq. 3) is determined by restrictions and resources. If a restriction is 1 or the resources are 0 , the autonomy is 0 and the potential option cannot be implemented.

Action $=f($ implementable options, motivations $)$

Implementable options $=$ potential options $*$ autonomy

Autonomy $=(1-$ restrictions $) *$ resources

$$
\begin{aligned}
& \text { Restrictions } \\
& \quad=f\left(T_{-} \text {TENANT }{ }^{a}, \text { CREEK, ETHNICITY }, \text { CROP }\right)
\end{aligned}
$$

$\begin{aligned} \text { Resources }= & I F\left(\text { assistance }+ \text { credit }+ \text { tenure } \_ \text {SIFMA }\right. \\ & - \text { investments }>=0), \text { resources }=1, \text { else } 0\end{aligned}$

Assistance $=($ MUNICIPALITY_ORG $+(A G E / 34)) / 3$

Credit $=f(C R O P)$

Tenure_SIFMA $=f(T E N U R E S I F M A, C R O P)$

Investment $=i n v \_b a s i c+i n v \_s l o p e$

inv_basic $=f(C R O P)$

inv_slope $=f(S L O P E, C R O P)$

${ }^{a}$ Variables in capitals

As described above the restrictions in this study are a function of tenancy, creek, ethnicity and crop (Eq. 4) (Table II). If the land manager is a tenant we consider only yellow corn to be an option. So, if the variable tenant is 1 , all land use types except corn were given value 1 . In the Eqs. 2 and 3 this leads to an autonomy of zero and therefore 
Table II Factors that Determine Autonomy Through Restrictions and Resources (Values in the Tables are Used in the Model)

\begin{tabular}{lllll}
\hline Variable & $\begin{array}{l}\text { Yellow } \\
\text { corn }\end{array}$ & $\begin{array}{l}\text { Wet } \\
\text { rice }\end{array}$ & Banana & $\begin{array}{l}\text { Fruit } \\
\text { trees }\end{array}$ \\
\hline $\begin{array}{l}\text { Restrictions } \\
\text { Tenure tenant=1 }\end{array}$ & 0 & 1 & 1 & 1 \\
$\quad$ Tenure tenant=0 & 0 & 0 & 0 & 0 \\
Creek=1 & 0 & 0 & 0 & 0 \\
Creek=0 & 0 & 1 & 0 & 0 \\
Eth. Ilocano and Ifugao & 0 & 0 & 0 & 0 \\
Eth. Ibanag, Kalinga & 0 & 1 & 0 & 0 \\
$\quad$ and Other & & & & \\
Resources & & & & \\
Credit & 1 & 0 & 0 & 0 \\
Tenure SIFMA=1 & 0 & 0 & 0 & 1 \\
Tenure SIFMA =0 & 0 & 0 & 0 & 0 \\
Investments & 1 & 1.2 & 0.3 & 1.5 \\
\hline
\end{tabular}

to a zero for the implementable options calculation, meaning the land use type is no option. Wet rice is only possible if a creek is nearby and if the field is cultivated by farmers of the ethnicity Ilocano or Ifugao. These relations are intuitively determined based on field experience and the interviews and are not fitted in any way.

In the model the resources assistance, credit and tenure SIFMA should cover the investments for a land use type to make this land use implementable (Eq. 5). The assistance depends on municipality of origin and average age of the household heads and is specified in Eq. 6. (For Ifugao the factor municipality of origin was fixed at 2 ). The equation is formulated in such a way that the result is centered around 34 years. This specific parameter was optimized, since no clear theoretical idea was available to determine the influence of age. As explained in the previous section, credit is 1 (possible) for yellow corn and 0 (not possible) for the other crops. The resource due to assistance by the NGO in the SIFMA areas is 1 for fruit trees (Table II). The investments consist of basic investments and investments due to slope (Eq. 9). The basic investments are defined as the basic investments necessary to start a new field for a specific land use type. The values of the basic investments (Table II) are relative to the initial investments for yellow

Table III Calculation of Investment Term for the Construction of Rice Terraces

\begin{tabular}{lll}
\hline Slope category & Days labor per ha* & Investment term for rice \\
\hline Slope1 & 52 & 0.00 \\
Slope2 & 292 & 0.36 \\
Slope3 & 716 & 1.00 \\
Slope4 & 2,209 & 3.25 \\
Slope5 & & $4.33^{* *}$ \\
\hline
\end{tabular}

* Source: Romero (personal communication) $(n=28)$

**Estimated by the authors
Table IV Maximum Benefit (in Ph. Pesos, Calculated from Field Data) per Land Use Type

\begin{tabular}{ll}
\hline Crop & Max_benefit \\
\hline Yellow corn & $22,435^{*}$ \\
Wet rice & $42,000^{*}$ \\
Banana & $21,213^{*}$ \\
Fruit trees & $32,230^{* *}$ \\
\hline
\end{tabular}

*Source: field data

**Source: Klein (2003)

corn, which were set on 1 . This relation was estimated by the authors based on field experience. The relation between slope and the investment necessary to build a rice terrace (Table III) was estimated according to the amount of labor necessary to build a terrace, which was calculated as an average from field observations. The extra investment due to slope was set on 1 for the terraces on slope category 3 and the other categories were estimated or calculated relative to this value.

The result of the model structure and the parameters is that corn is possible for all farmers because investments can be covered by credits, banana is also possible for all farmers because the initial investments are low and fruit trees are possible for people who have a SIFMA lot. Initially, the calculation resulted in no possibilities for wet rice, because of too high initial investments. Since rice does occur in the area this rule was relaxed a little. This can be justified by the fact that rice fields are usually smaller than a hectare and the calculation is per hectare and therefore initial investments are smaller in reality than the calculated investments.

The right branch of the AiC model (Fig. 1) deals with the motivations. The motivations (as interpreted) consist of objectified motivations multiplied with a factor for the preferences (Eq. 12). In this case the objectified motivations are expressed in Philippine Pesos and consist of the maximum benefit, a slope factor, a risk factor and transportation cost. The maximum benefit is expressed in Table IV. The maximum benefit is multiplied by a yield factor depending on slope for corn (Table V) and an average yield reducing factor depending on estimated risks for all land use types (Table VI). The high typhoon risk for banana is related to the fact that the banana is not pro-

Table V Calculation of Slope Factor for Yellow Corn

\begin{tabular}{lll}
\hline Slope category & Average yield $(\mathrm{kg} / \mathrm{ha})$ & Slope_factor \\
\hline Slope1 & 3,581 & 1.00 \\
Slope2 & 3,829 & 1.07 \\
Slope3 & 3,070 & 0.86 \\
Slope 4 & no data & $0.50^{*}$ \\
Slope5 & no data & $0.20^{*}$
\end{tabular}

*Estimated by the authors; other data based on field observations $(n=37)$ 
Table VI Risk Factors of Crops

\begin{tabular}{lllll}
\hline Risk/Crop & Yellow Corn & Wet rice & Banana & Fruit trees \\
\hline Typhoon & 0.10 & 0.05 & 0.20 & 0.10 \\
Drought & 0.10 & 0.05 & 0.02 & 0.02 \\
BBTV & 0.00 & 0.00 & 0.10 & 0.00 \\
Risk total & 0.20 & 0.10 & 0.32 & 0.12 \\
\hline
\end{tabular}

N.B. All numbers are estimated by the authors

ductive for 1-1.5 years after a typhoon, while other crops can be replanted and productive several months after destruction. The transportation costs are computed according to Verburg et al. (2004a). Traveling distance to the plot ('plot distance') was translated into monetary costs. For wet rice transportation costs were only based on costs from the residence to the field, since the product is not marketed, and for the other crops the calculation is a combination of costs from field to residence and from residence to the town of San Mariano. The preferences (Table VII) based on ethnicity were quantified by the authors based on qualitative descriptions by the farmers. It may be noted that in this model the effect of the preference for rice cultivation is cancelled out by the much higher net benefit of rice compared to the other crops, so differences in preference do not change the prediction of rice.

Motivations $=$ objectified motivations $*$ preferences

\section{Objectified motivations (net benefit)}

$$
=\max \_ \text {benefit } * \text { slope_fact } *(1-\text { risk })-t r_{-} \text {costs }
$$

Max_benefit $=f(C R O P)$

Slope_fact $=f(S L O P E, C R O P)$

Risk $=f(C R O P)$

$T r_{-}$costs $=f\left(T R \_C O S T, P L O T \_D I S T A N C E, C R O P\right)$

Preferences $=f($ ETHNICITY, CROP $)$

Table VII Preference Factors Based on Ethnicity

\begin{tabular}{lllll}
\hline Ethnicity/crop & Yellow corn & Wet rice & Banana & Fruit trees \\
\hline Ifugao & 0.9 & 1.2 & 1 & 1 \\
Ibanag & 1.2 & 0.9 & 1 & 1 \\
Ilocano & 1 & 1.2 & 1 & 1 \\
Kalinga & 1 & 1 & 1 & 1 \\
Other & 1 & 1 & 1 & 1 \\
\hline
\end{tabular}

N.B. All numbers are estimated by the authors
Table VIII Summary of the Motivational Value for all Fields per Land Use Type

\begin{tabular}{lll}
\hline Land use & Average (Peso/ha) & St.dev \\
\hline Yellow corn & 14,239 & 3,834 \\
Wet rice & 40,694 & 5,181 \\
Banana & 12,474 & 672 \\
Fruit trees & 23,313 & 1,739 \\
\hline
\end{tabular}

The objectified motivations with the interpretations combine into the motivations (as perceived and valued) for each field for all four crops. These motivations are summarized in Table VIII. Calculated per hectare the cultivation of wet rice is by far the most profitable followed by fruit trees. The benefits from corn and banana are very similar.

\section{Model Results}

The Inductive (Multinomial Regression) Model

The inductive model (Table IX) shows the estimated parameters of wet rice, banana and fruit trees in relation to yellow corn, which is the reference category. The estimated coefficients should be interpreted relative to this category. For example, one unit increase in the explanatory variable creek will increase the $\ln \left(\mathrm{P}_{\text {wet rice }} / \mathrm{P}_{\text {yellow corn }}\right)$ with 1.988. In multinomial regression analysis the interpretation of the estimated coefficients is not completely straightforward, because the coefficients only tell us the relation between one land use category and the reference category. This complicates direct comparison of the inductive model with the deductive model.

The cross-tabulation (Table X) shows the number of observations modeled correctly (the bold diagonal figures).

Table IX The Multinomial Regression Model

\begin{tabular}{llll}
\hline Variables & $\begin{array}{l}\text { Wet rice } \\
\mathrm{b}\end{array}$ & $\begin{array}{l}\text { Banana } \\
\mathrm{b}\end{array}$ & $\begin{array}{l}\text { Fruit trees } \\
\mathrm{b}\end{array}$ \\
\hline Intercept & -3.182 & $-9.936^{* * *}$ & $-11.420^{* * *}$ \\
Slope & $-1.302^{* *}$ & $2.224^{* * *}$ & $1.628^{* * *}$ \\
Ethnicity Ifugao male & $2.631^{*}$ & -0.295 & -1.588 \\
Ethnicity Ilocano male & $1.678^{*}$ & 0.380 & -0.131 \\
Municipality of origin & -0.668 & -0.097 & -0.402 \\
Creek & $1.988^{* * *}$ & 0.013 & 0.502 \\
Plot distance & -0.008 & 0.008 & $0.016^{*}$ \\
Transportation cost & $0.051^{*}$ & $0.065^{* *}$ & 0.050 \\
Average age & 0.011 & 0.037 & 0.067 \\
Tenure tenant & 0.084 & -0.921 & 0.159 \\
Tenure SIFMA & -0.908 & 0.749 & $3.931^{*}$ \\
\hline
\end{tabular}

${ }^{*} p<0.05, * * p<0.01, * * * p<0.001$ 
Table X Observed Land Use vs. Modeled Land Use of the Inductive (Regression) Model

\begin{tabular}{lllllll}
\hline \multicolumn{7}{c}{ Predicted land use type } \\
\cline { 2 - 7 } & $\begin{array}{l}\text { Wet } \\
\text { rice }\end{array}$ & Banana & $\begin{array}{c}\text { Fruit } \\
\text { trees }\end{array}$ & $\begin{array}{l}\text { Yellow } \\
\text { corn }\end{array}$ & Total & $\begin{array}{l}\% \\
\text { Correct }\end{array}$ \\
\hline $\begin{array}{l}\text { Inductive (regression) } \\
\text { Observed }\end{array}$ & & & & & \\
Land use & & & & & & \\
type & & & & & & \\
Wet rice & $\mathbf{1 8}$ & 1 & 1 & 16 & 36 & 50.0 \\
Banana & 0 & $\mathbf{4 2}$ & 3 & 19 & 64 & 65.6 \\
Fruit trees & 0 & 5 & $\mathbf{6}$ & 3 & 14 & 42.9 \\
Yellow & 4 & 11 & 0 & $\mathbf{1 4 3}$ & 158 & 90.5 \\
$\quad$ corn & & & & & & \\
Total & 22 & 59 & 10 & 181 & 272 & 76.8 \\
\hline
\end{tabular}

Yellow corn was fitted very well (91\%), banana was fitted reasonably well $(66 \%)$, and wet rice $(50 \%)$ and fruit trees (43\%) were fitted somewhat weakly. In total, the multinomial regression model fitted a total of 209 out of $272(77 \%)$ observations correctly. A test was performed to determine the extent the observed and modeled land use distributions are alike. The Chi-square statistic of this test is significant at the 0.0001 level. The kappa statistic, indicating the proportion of agreement after chance has been excluded, is 0.579 .

\section{The Deductive (AiC) Model}

The results of the deductive model (Table XI) are largely the same as the results of the inductive model. Wet rice is predicted better than in the multinomial model. For the other land use types the deductive model performed slightly less well. The model was able to predict $70 \%$ of the occurring land uses of a dataset of 272 fields. The Chisquare statistic is significant $(p<0.0001)$ and the kappa statistic is 0.471 . The kappa statistics of the two models are not significantly different $(p<0.05)$ (Couto, 2003).

\section{Discussion and Conclusions}

\section{Factors of Land Use Change}

The AiC framework is designed to incorporate relevant factors from all scientific disciplines in a balanced manner. Using the deeper analysis of the AiC framework as a template for the deductive model, we were able to incorporate variables from various disciplines, including geographic (e.g. slope, presence of creek), economic (e.g. investments, net benefit), social (age, municipality of origin), anthropo- logical (ethnicity), and policy (SIFMA) variables. The same factors are incorporated in the inductive model and in that respect both models are equally multi-disciplinary, 'integrated' models. The factors comprise a good many of those listed in overviews of driving factors by Geist and Lambin (2002) and Lambin et al. (2003), even though we have focused only on simple crop choices. Since we have not compared land use in two or more points in time, our factors are explanatory factors rather than dynamic 'drivers' of land us change, formally. Predictions of the effect of incremental changes in factors may be derived from both models, however (as in Nelson et al. 2001). In the sense of factors and predictions, therefore, the present study is comparable to mainstream land use studies.

Differences Between Inductive and Deductive Approaches

The results of the inductive and deductive approach look much alike, on the surface, because the inductive model fitted $77 \%$ of the observations correctly, and the deductive model predicted $70 \%$ of the observations correctly. On a deeper level, however, important differences between the two models present themselves. Deductive approaches, starting out from theory and maintaining theoretical structures, feed better back into theory development than inductive studies tend to do. The present study, for instance, is a true verification of broad rational choice theory of land use, expressed in the structure of Action-in-Context's decision model. The Popperian critique here would be, of course, that this is no surprise because one should aim to verify unlikely structures, or to falsify the likely ones. Nevertheless, an entry to the theory level it is and once there, progress may be pursued in many directions, including the testing of less likely actor models or spatially explicit neo-Thünian theory of moving land use frontiers (e.g.

Table XI Observed Land Use vs. Modeled Land Use of the Deductive (AiC) Model

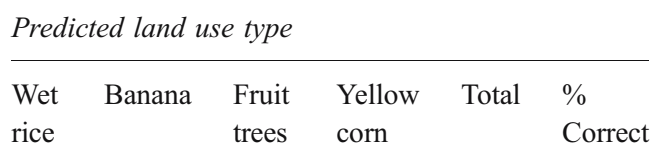

Deductive (AiC) model

Observed

Land use

type

\begin{tabular}{lllllll} 
Wet rice & $\mathbf{2 1}$ & 1 & 1 & 13 & 36 & 58.3 \\
Banana & 2 & $\mathbf{3 1}$ & 5 & 26 & 64 & 48.4 \\
Fruit trees & 1 & 3 & $\mathbf{5}$ & 5 & 14 & 35.7 \\
Yellow & 18 & 5 & 1 & $\mathbf{1 3 4}$ & 158 & 84.8 \\
$\quad$ corn & & & & & & \\
Total & 42 & 40 & 12 & 178 & 272 & 70.2 \\
\hline
\end{tabular}


De Groot, 1999). The model structure may also be expanded easily to include multi-level causal influences on the region's land use, for instance through AiC's actors field, see Verburg et al. (2003).

A second advantage of deductive work is that, as it tests full causal structures rather than separate causal factors, a much better grip on causality is established. Two specific aspects of this characteristic stand out from the present study. (1) Any inductive model, working as it does from the data 'upward,' can only fit for variables that vary across the dataset. The influence of all factors that are constant across the dataset, such as in our case the market price of corn, end up, implicitly, in the intercept $\left(\beta_{0}\right)$. Therefore, it is less straightforward to predict the amount of land use change for a change in one of the factors accumulated in the intercept. A deductive model, however, allows the inclusion of all factors assumed to have causal influence (hence relevant for explanations as well as policy-oriented predictions), such as changes in price. (2) For the same reason of testing full structure rather than factors, deductive models are able to handle new phenomena, assuming that they do not alter the model structure. In the study area, for instance, cassava may be an alternative cash crop. The inductive model cannot handle this, because cassava is new, hence absent from the dataset and therefore from the model. In order to make a prediction of the region's response to cassava by way of the deductive model we do need general cassava production data such as its price, productivity, position in cultural preferences, accompanying credit scheme, etc., but once we have these, a prediction is produced and various policy scenarios may be studied.

\section{Reaping the Benefits of Combining Inductive} and Deductive Approaches

Research programs often tend toward a certain development on the deduction/induction ladder. For example, starting out with a more or less extreme data mining (rung 1), the selection and shaping of causal factors may become led more by insight (rung 2) and researchers may end up in studies more consciously and fully in discussion with theory (rung 3). In fact, quite a number of inductively acquired insights into our field research region, gathered by previous studies, informal discussions and visits, interviews and observations, underlie our own deductive model. In other words, we sojourned long on rungs 1 and 2 before our deductive jump to rung 5 in the present paper. For the sake of clear-cut illustration we refrained here from what would have been the natural follow-up of our strictly deductive approach, namely, to better calibrate and fit our model parameters on reality, i.e. move one level down to rung 4, searching to reach a better fit than the $70 \%$ of the strictly deductive model. After that, we could even have begun to adapt our whole model structure in discussion with theory and field realities, thereby arriving at rung 3 .

Overall, then, the most effective way to reap the benefits of more deductive work does not seem to be to rigidly 'go deductive' and stay there. Rather, the message should be that research will profit most from a consciousness of the whole spectrum between the inductive and deductive extremes, an awareness of the advantages of deductive approaches versus the currently dominant inductive research routines, and then a search for the most fertile sequences and interactions between inductive and deductive work. This then is the invitation meant to emanate from the present paper to the scientific community of land use change research.

Acknowledgements The authors would like to acknowledge WOTRO-NWO for the funding of the research on which this paper is based. Special thanks go to Noel Perez, who was the research assistant for this project during the fieldwork in the Philippines, and Marino Romero for their contributions to this paper. We want to thank the CVPED (Cagayan Valley Programme on Environment and Development) staff in Cabagan and the LGU (Local Government Unit) of San Mariano for their support.

\section{References}

Bebbington, A. (1999). Capitals and Capabilities: A Framework for Analyzing Peasant Viability, Rural Livelihoods and Poverty. World Development 27 (12): 2021-2044.

Blaikie, P. (1985). The Political Economy of Soil Erosion in Developing Countries. Longman, Harlow.

Brox, O. (1990). The Common Property Theory-Epistemological Status and Analytical Utility. Human Organization 49 (3): $227-$ 235.

Couto, P. 2003. Assessing the Accuracy of Spatial Simulation Models. Ecological Modeling 167: 181-198.

De Groot, W. T. (1992). Environmental Science Theory; Concepts and Methods in a One-world, Problem Oriented Paradigm. Elsevier Science Publishers, Amsterdam.

De Groot, W. T. (1999). Van vriend naar vijand naar verslagene en verder. Een evolutionair perspectief op de verhouding tussen ment en natuur. Nijmegen University Press, Nijmegen, The Netherlands.

Elster, J. (1989). Nuts and Bolts for the Social Sciences. Cambridge University Press, Cambridge.

Geist, H. J., and Lambin, E. F. (2002). Proximate Causes and Underlying Driving Forces of Tropical Deforestation. BioScience 52 (2): $143-150$.

Huigen, M. G. A. (2004). First Principles of the MameLuke Multiactor Modelling Framework for Land use Change, Illustrated with a Philippine Case Study. Journal of Environmental Management 72 (1-2): 5-21.

Klein, M. (2003). Introducing fruit-trees at smallholder's farmers: potential and constraints. (Environment and development student report, nr. 160). Leiden; Cabagan: Leiden University, CML; Isabela State University, CVPED.

Lambin, E. F., Geist, H. J., and Lepers, E. (2003). Dynamics of Landuse and Land-cover Change in Tropical Regions. Annual Review of Environment and Resources 28: 205-241. 
Liao, S. (2003). Knowledge Management Technologies and Applications-Literature Review from 1995 to 2002. Expert System with Applications 25: 155-164.

Moonen, E. (2002). Improving Your Land...: From 'swidden' to 'ricepaddy' in the Forest-frontier of the Sierra Madre Mountains of Luzon, the Philippines. Centre of Environmental Science (CML), Leiden, The Netherlands.

Müller, D., and Zeller, M. (2002). Land use Dynamics in the Central Highlands of Vietnam: A Spatial Model Combining Village Survey Data with Satellite Imagery Interpretation. Agricultural Economics 27 (3): 333-354.

Nelson G. C., Harris V., and Stone S. W. (2001). Deforestation, Land Use, and Property Rights: Empirical Evidence from Darien, Panama. Land Economics 77 (2): 187-205.

Nelson, G. C., De Pinto, A., Harris, V., and Stone, S. (2004). Land Use and Road Improvements: A Spatial Perspective. International Regional Science Review 27 (3): 297-325.

Ostrom, E. (1990). Governing the Commons: The Evolution of Institutions for Collective Action. Cambridge University Press, Cambridge.

Overmars, K. P., and Verburg, P. H. (2005). Analysis of Land Use Drivers at the Watershed and Household Level: Linking Two Paradigms at the Philippine Forest Fringe. International Journal of Geographical Information Science 19 (2): 125-152.

Perz, S. G., and Walker, R. T. (2002). Household Life Cycles and Secondary Forest Cover Among Small Farm Colonists in the Amazon. World Development 30 (6): 1009-1027.

Popper, K. R. (1963). Conjectures and Refutations: The Growth of Scientific Knowledge. Routledge and Kegan Paul, London.

Serneels, S., and Lambin, E. F. (2001). Proximate Causes of Landuse Change in Narok District, Kenya: A Spatial Statistical Model. Agriculture, Ecosystems and Environment 85 (1-3): 6581.

Tadepally, H. (1999). Neglect and Restoration of Minor Irrigation Tanks in Krishna District, India : A Local-level Case Study of Motivation, Capacity and Partnership Interventions. Nuzvid, Maruthibala Publishers.
Valmayor, R. V., Jones, D. R., Subijanto, Polprasid, P., and Jamaluddin, S. H. (1990). Bananas \& Plantains in Southeast Asia, INIBAP, Montpellier.

Van den Top, G. M. (1998). The social dynamics of deforestation in the Sierra Madre, Philippines. PhD. Thesis, Centre of Environmental Science, Leiden University, Leiden, The Netherlands.

Vayda, A. P. (1983). Progressive Contextualization: Methods for Research in Human Ecology. Human Ecology 11 (3): 265-281.

Vayda, A. P., and Walters, B. B. (1999). Against Political Ecology. Human Ecology 27 (1): 167-179.

Verburg, P. H., De Groot, W. T., and Veldkamp, A. (2003). Methodology for Multi-scale Land-use Modelling: Concepts and Challenges. In Dolman, A. J., Verhagen, A., and Rovers, C. A. (eds.), Global Environmental Change and Land Use. Kluwer Publishers, Dordrecht/Boston/London.

Verburg, P. H., Overmars, K. P., and Witte, N. (2004a). Accessibility and Land use Patterns at the Forest Fringe in the Northeastern Part of the Philippines. The Geographical Journal 170 (3): 238-255.

Verburg, P. H., Schot, P., Dijst, M., and Veldkamp, A. (2004b). Land use Change Modelling: Current Practice and Research Priorities. GeoJournal 61 (4): 309-324.

Walker, R. (2004). Theorizing Land-cover and Land-use Change: The Case of Tropical Deforestation. International Regional Science Review 27 (3): 247-270.

Walker, R., and Solecki, W. (2004). Theorizing Land-cover and Landuse Change: The Case of the Florida Everglades and its Degradation. Annals of the Association of American Geographers 94 (2): 311-328.

Walker, R., Perz, S., Caldas, M., and Silva, L. G. T. (2002). Land use and Land Cover Change in Forest Frontiers: The Role of Household Life Cycles. International Regional Science Review 25 (2): 169-199.

Zuiderwijk, A. B. (1998). Farming Gently Farming Fast. Migration, Incorporation and Agricultural Change in the Mandara Mountains of Northern Cameroon. $\mathrm{PhD}$ dissertation, Leiden University, Leiden, The Netherlands. 